\title{
INTERVENÇÕES UTILIZANDO O MODELO TRANSTEORÉTICO PARA A ATIVIDADE FÍSICA: ESTUDO BIBLIOMÉTRICO
}

\section{INTERVENTIONS USING THE TRANSTHEORETICAL MODEL FOR PHYSICAL ACTIVITY: BIBLIOMETRIC STUDYINTERVENÇÕES}

\author{
Hector Luiz Rodrigues Munaro ${ }^{1}$, Suziane de Almeida Pereira Munaro \\ Universidade Estadual do Sudoeste da Bahia $-\mathrm{UESB}^{1}$; Núcleo Regional de Educação da Bahia - NRE $22^{2}$
}

\begin{abstract}
There is a shortage of bibliometric studies that demonstrated the main school-based interventions among adolescents with physical activity as the main outcome. The objective of this study was to identify, through bibliometrics, an exploratory-descriptive technique, the national and international scientific productions. As a method, bibliometrics was used through the process of bibliographic revision called Knowledge Development Process - Construtivist (ProKnow-C). Scientific articles were analyzed in the Capes Periodical Portal, produced between 2003 and 2013. The Bibliographic Portfolio was composed of eleven articles that aligned with the theme "School-Based Interventions Using the Transtheoretic Model for Physical Activity". It was identifying the main authors, articles, periodicals, impact factor and keywords, then performed systemic analysis. Two studies of the same author have obtained the great part of quotations, only one periodical published more of an article related to the subject and that no national study in the search was found. It was evidenced that most of the studies used interventions between three and six months in duration, with adequate statistics, showing different results in relation to the effectiveness of the model.
\end{abstract}

Key words: Intervention studies, Bibliometrics, Motor Activity, Adolescents Behaviour

\section{Resumo}

Há escassez de estudos bibliométricos que demonstre as principais intervenções de base escolar entre os adolescentes com a atividade física como desfecho principal._o objetivo deste estudo foi identificar, através da bibliometria, uma técnica exploratória-descritiva, estas produções científicas nacionais e internacionais. Como método, utilizou-se a bibliometria por meio do processo de revisão bibliográfica denominado Knowledge Development Process - Construtivist (ProKnow-C). Foram analisados artigos científicos, no Portal de Periódicos Capes, produzidos entre 2003 e 2013. O Portfólio Bibliográfico foi composto por onze artigos que se alinharam ao tema "Intervenções de base escolar utilizando o Modelo Transteorético para a Atividade Física". Foi identificando os principais autores, artigos, periódicos, fator de impacto $e$ palavras-chave, em seguida realizada análise sistêmica. Dois estudos de uma mesma autora obtiveram a maior parte das citações, apenas um periódico publicou mais de um artigo relacionado ao tema e que não foi encontrado nenhum estudo nacional na busca. Ficou evidenciado que a maioria dos estudos utilizaram intervenções entre três e seis meses de duração, com estatística adequada, mostrando resultados diferentes em relação à efetividade do modelo.

Palavras chave: Estudos de intervenção, Bibliometria, Atividade Física, Comportamento do Adolescente 
Introdução

Os determinantes comportamentais parecem influenciar diretamente nos fatores de risco à saúde de adolescentes, uma vez que esta fase é caracterizada por diversas transformações biológicas, cognitivas e comportamentais, e as influencias de pais, amigos e professores, podem facilitar a implementação de um estilo de vida mais saudável, evitando-se o acometimento de doenças na vida adulta ${ }^{1}$. Assim, o Modelo Transteorético (MT), através dos constructos dos Estágios de Mudança de Comportamento (EMC), pode ser utilizado como delineamento de intervenções para estabelecer a relação comportamental entre os fatores de riscos e as barreiras percebidas para a efetiva mudança ${ }^{2}$.

Intervenções que utilizam o MT, através dos EMC, são bastante frequentes em temáticas como alimentação, fumantes, comportamentos de risco e sexo sem segurança ${ }^{2}$. Parte destes estudos é de natureza clínica, utilizando as estratégias de cada estágio de mudança de comportamento com vista às possíveis mudanças no curto e médio prazos, dependendo do estágio em que se encontram ${ }^{3}$. Esta teoria possui esta denominação, pois se utiliza da mudança de comportamento baseada nos processos integrativos e princípios de diversas teorias de intervenção.

O constructo deste modelo pressupõe que o processo de mudança aconteça em estágios, com o indivíduo atingindo determinado estágio ou recaindo para estágios anteriores. Os estágios propostos são: a) pré-contemplação; b) contemplação; c) preparação; d) ação; e, e) manutenção ${ }^{2}$. Para que as intervenções utilizando este modelo possam ser efetivas em determinado espaço temporal, é necessário que se siga alguns pressupostos empíricos, já que a mudança é algo pessoal. Desta forma, determinados processos de mudança, balanços de decisão e auto-eficácia, devem ser considerados para se fomentar uma determinada intervenção, independente de qual comportamento se propõe a modificar ${ }^{3}$.

$\mathrm{Na}$ área de atividade física relacionada à saúde de adolescentes, observamos predominância de estudos epidemiológicos transversais utilizando este modelo apenas para a identificação de prevalência de inatividade física e quais fatores associados ${ }^{4,6}$. Porém, os estudos de intervenção de base escolar, utilizando as estratégias do Modelo Transteorético, ainda são escassos.

Dos estudos selecionados na literatura para se demonstrar intervenções utilizando o MT, destaca-se uma revisão sistemática com metaanálise ${ }^{7}$, realizada com artigos de países da Europa e Estados Unidos, onde menos da metade dos 14 estudos encontrados, utilizaram modelos de intervenção baseados em alguma teoria comportamental. Mesmo assim, aqueles que utilizaram, contribuíram para o aumento no tempo gasto em atividade física em relação aos grupos controle. Já em revisão sistemática, realizada na America Latina ${ }^{8}$, os autores identificaram, dentre os 29 artigos encontrados na busca, que apenas 12 utilizaram o modelo transteorético, mostrando resultados divergentes em relação à efetividade destas intervenções.

Para subsidiar pesquisadores acerca dos principais estudos em determinada área de conhecimento, nos últimos anos, têm se intensificado os estudos bibliométricos. Alguns estudos ${ }^{9,10}$ utilizam técnicas exploratóriodescritiva para identificar, entre outras demandas já disponíveis em revisões sistemáticas, o aprofundamento de determinado tema pelo pesquisador, bem como identificar onde se encontram os estudos (através de análise quantitativa de citações, fator de impacto, palavras-chave mais utilizadas e ano de publicação) com o objetivo de identificar os pesquisadores e periódicos com maior reconhecimento na literatura de determinada área de conhecimento ou tema proposto ${ }^{11}$, além de realizar uma análise sistêmica dos artigos e propor novas temáticas ou constructos ${ }^{12}$.

Não foram encontradas, pelos pesquisadores, até o momento de conclusão deste artigo, estudos bibliométricos acerca de intervenções utilizando o modelo transteorético para atividade física em adolescentes, justificando esta pesquisa.

Assim, justifica-se o presente estudo, onde o objetivo foi levantar através da técnica de bibliometria, utilizando um método exploratóriodescritivo, as produções científicas, sobre intervenções de base escolar, com adolescentes, utilizando o modelo transteorético tendo como desfecho a atividade física.

\section{Metodologia}

Estudo exploratório-descritivo, que buscou, em bases de dados, artigos científicos com a temática "Intervenções em Atividade Física utilizando o Modelo Trasnteorético em adolescentes". Através de um Portfólio Bibliográfico $^{9-12}$, que consiste em um quadro das 
referências bibliográficas alcançadas na busca, descreveram-se as características dos artigos, periódicos que mais publicaram sobre o tema, os principais autores, os artigos com maior reconhecimento, segundo critérios do método ${ }^{9}$, através do número de citações e fator de impacto dos periódicos, sobre o tema e uma análise sistêmica através de alguns critérios (lentes) previamente adotados.

Foi utilizado o processo de revisão bibliográfica denominada Knowledge Development Process- Construtivist (ProKnowC) ${ }^{9}$, desenvolvido por pesquisadores da
Universidade Federal de Santa Catarina. Consiste em um instrumento que é apresentado em forma descritiva, simples, transparente e sistematizado, sendo os critérios de análise integrados, e composto por quatro etapas: 1) seleção de um conjunto de artigos sobre o tema da pesquisa (portifólio bibliográfico); 2) análise bibliométrica dos artigos; 3) análise sistêmica; e, 4) definição da pergunta de pesquisa e objetivo de pesquisa $^{10,11}$. No presente estudo, foram utilizadas as etapas de 1 a 3 , conforme apresentado na Figura 1, destacadas com o círculo.

Figura 1 - Etapas do Método PróKnown-C.

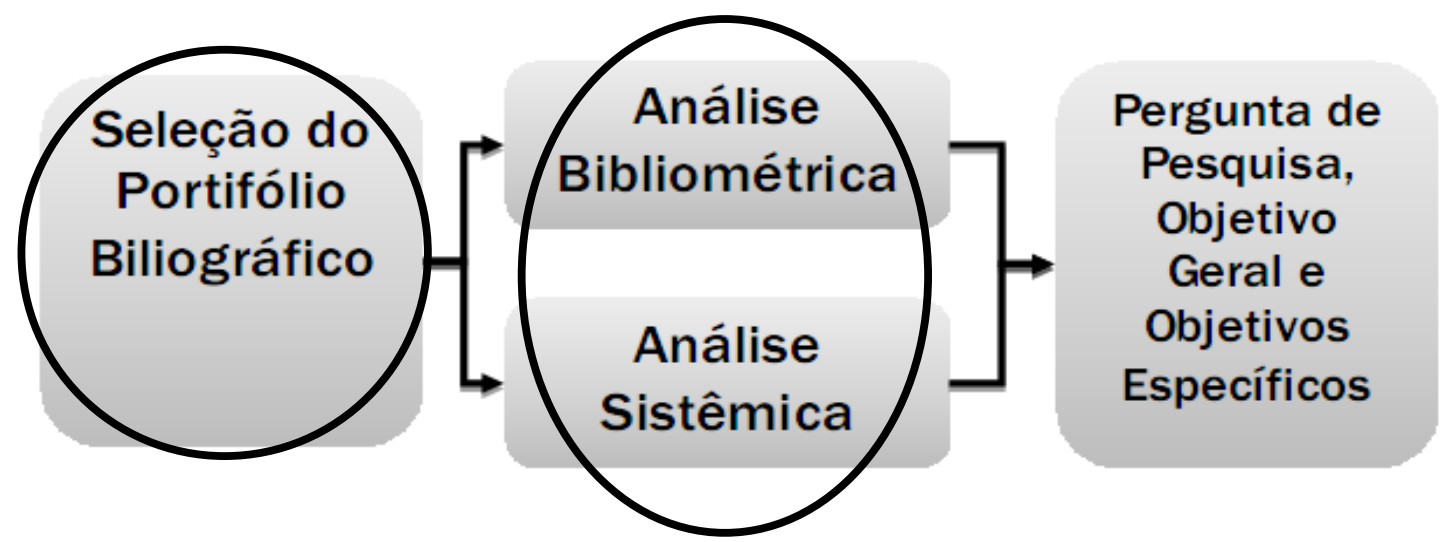

Fonte: Adaptado de Vilela, $2012^{11}$.

A seleção do Portifólio Bibliográfico foi realizada nas seguintes etapas: 1)seleção dos artigos nas bases de dados, compondo o Banco de Artigos Brutos; e, 2)filtragem dos artigos selecionados com base no alinhamento com o tema da pesquisa, definidos nas ferramentas de busca, realizadas por dois pesquisadores ${ }^{10,11}$.

$\mathrm{Na}$ fase de confecção do Banco de Artigos Brutos, foram seguidas as seguintes etapas: 1 ) definição das palavras-chave; 2) definição da base de dados de busca;3) busca dos artigos com as palavras-chave; e, 4) realização de teste de aderência das palavras-chave ${ }^{10,11}$.

A base de dados utilizada foi a do Portal de Periódicos Capes, justificada por reunir grande parte das bases de dados nacionais e internacionais. Foram determinadas as palavraschave de acordo com tema escolhido para o estudo. A busca dos artigos foi realizada no período de setembro e outubro de 2014. Utilizou-se o espaço temporal de publicação compreendido entre 1980 e 2012. O primeiro período deve-se ao início do desenvolvimento dos estudos acerca do Modelo Transteorético, conforme descrito pelos idealizadores do modelo $^{2}$, e o final justifica-se uma vez que estudos publicados a menos de dois anos podem não ter, ainda, uma quantidade de citações relevantes, de acordo com o método ${ }^{9}$, apesar de serem mais atuais. Após esta etapa, foi realizada uma filtragem dos artigos brutos considerando as etapas: 1) se os artigos não estão repetidos (redundância); 2) os títulos dos artigos estão alinhados com o tema da pesquisa; 3) os artigos possuem reconhecimento científico; 4) os resumos dos artigos estão alinhados com o tema da pesquisa; e, 5) se o texto integral dos artigos está alinhado com o tema da pesquisa ${ }^{9-12}$, sendo esta etapa realizada por dois pesquisadores.

Foi realizada a análise bibliométrica do Portfólio Bibliográfico desenvolvida em quatro etapas: 1) estimativa do grau de relevância dos periódicos; 2) estimativa do reconhecimento científico dos artigos; 3) estimativa do grau de relevância dos autores; e, 4) estimativa das palavras-chave mais utilizadas ${ }^{10,12}$.

Para a confecção do Banco de Artigos Brutos foram utilizadas as seguintes palavras-chave em língua inglesa: Transtheoretical Model, Stage of Change, Interventions, Youth, Adolescents, School-based e Motor Activity. Foram feitas combinações com os operadores boleanos AND e 
OR.

Definidas as palavras-chave e suas combinações, foi realizada a busca inicial no Portal de Periódicos Capes, tendo sido encontrados 1.463 estudos. Logo em seguida, foi realizado teste de aderência, que tem como propósito identificar se as palavras-chave de uma amostra dos estudos selecionados possuem congruência com os definidos no estudo bibliométrico. Para tanto, foram selecionados, aleatoriamente, dois estudos dentre os encontrados e realizado o teste por algumas vezes, até que fossem encontrados estudos que atendessem aos critérios de alinhamento.

Nesta etapa, foram separados oito estudos pois não possuíam pelo menos três palavraschave que coincidiam com as sete determinadas e suas combinações definidas para a busca ${ }^{10}$. No entanto, não foram totalmente descartados, já que mesmo não atendendo ao critério, após a leitura dos resumos, poderiam ser incluídos no banco de artigos.

$\mathrm{Na}$ Figura 2 destaca-se o processo de seleção da confecção do Banco de Artigos Brutos e o Portfólio Bibliográfico.

Figura 2 - Processo de Confecção do Banco de Artigos Brutos e Portfólio Bibliográfico.

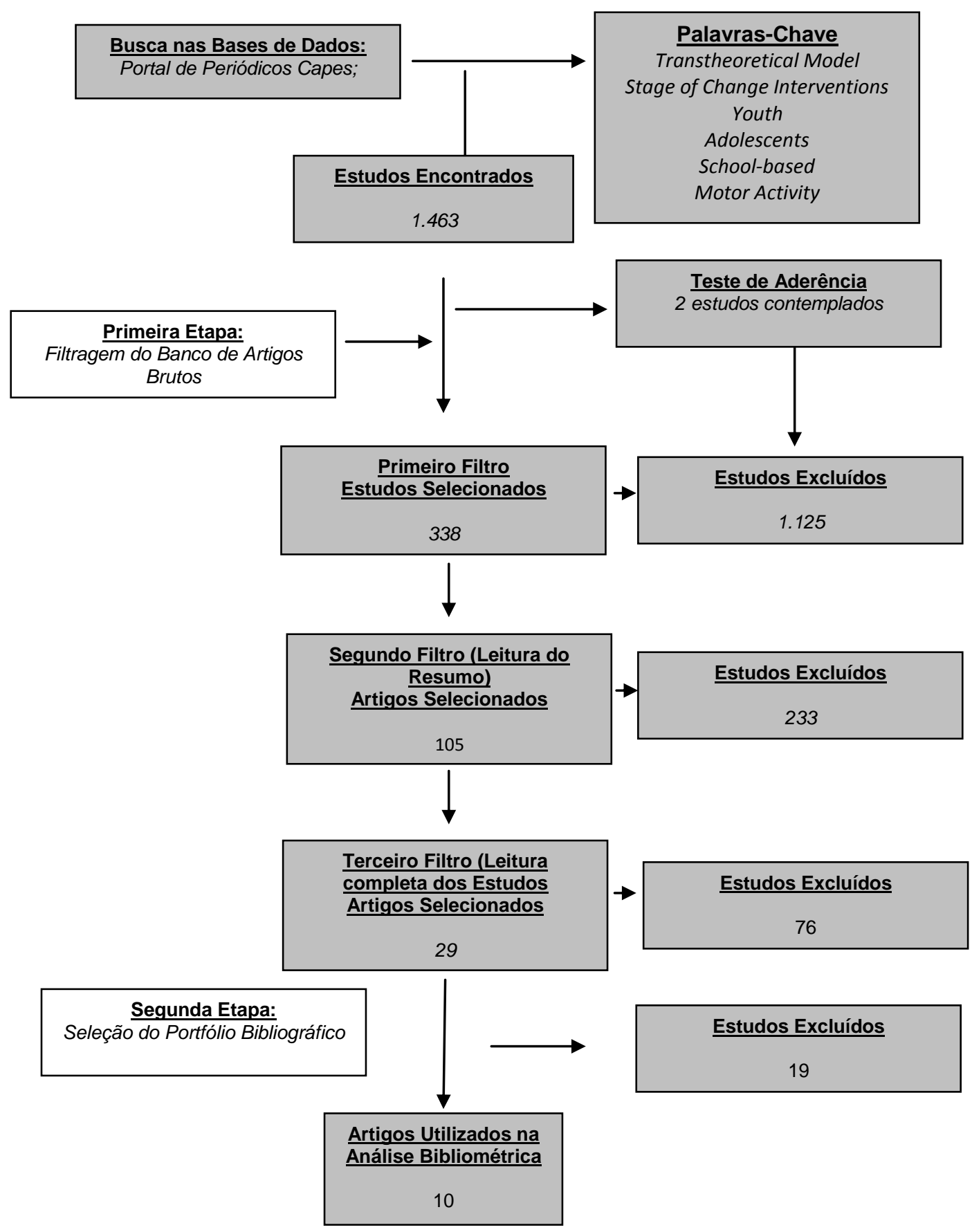




\section{Resultados}

$\mathrm{Na}$ primeira filtragem da confecção do Banco de Artigos Brutos, após as referências serem exportadas para a ferramenta EndNote ${ }^{13}$, foram excluídos 1.112 estudos por não possuírem o título dos estudos alinhado ao tema da pesquisa. Eram teses ou dissertações, muitos repetidos (redundância) e foram excluídos no teste de aderência ou não estavam disponíveis para download gratuitamente.

Após este procedimento, foram lidos e analisados, pelos dois autores, novamente os títulos e os resumos de 338 estudos selecionados após o primeiro filtro e quando havia divergência estes estudos eram novamente lidos.

Assim, 234 foram eliminados por não se alinharem ao tema da pesquisa, incluindo-se oito artigos que haviam sido separados no teste de aderência. Nesta etapa, foram incluídos apenas os artigos publicados em periódicos que eram revisados por pares (controle de qualidade).

Para a leitura completa dos artigos, foram selecionados 105 no segundo filtro. Após a leitura completa, foram selecionados, no terceiro filtro, na segunda etapa, apenas 25 artigos que possuíam o resumo e o texto completo alinhados ao tema de pesquisa.

Foi realizado, ainda, um teste de reconhecimento científico dos estudos através da análise de citações dos artigos, utilizando o Google Scholar como ferramenta. Desta forma, foram excluídos 15 artigos que possuíam menos de uma citação e aqueles que foram publicados após 2013.

Ao final, foram selecionados para comporem o Portfólio Bibliográfico, 11 estudos, os quais, somadas, continham 411 referências. Para garantir uma busca mais efetiva, as referências foram incluídas em uma nova etapa para verificar se haviam estudos que pudessem compor o Portfólio. Nesta etapa, apenas um estudo foi incluído ao final.

A Tabela 1 apresenta o Portfólio Bibliográfico com os respectivos artigos que o mesmo é composto.

Seguindo a análise do Portfólio Bibliográfico (Tabela1), os artigos mais citados, independente do ano de publicação, foram: New Moves: A school-based obesity prevention program for adolescent girls e Factors associated with changes in physical activity: a cohort study of inactive adolescent girls, com 319 e 253 citações, respectivamente, ambos publicados em 2003. O que chama atenção é que ambos foram produzidos pela mesma autora (considerando apenas o primeiro autor) e abordaram programas de intervenção entre as moças.

Quando analisados os autores, considerando apenas o primeiro autor, observouse que houve um autor de destaque (Leen Haerens) com três artigos encontrados, já outros autores tiveram dois artigos selecionados, sendo eles: Dianne Neumark-Sztainer, Parvaneh Taymoori e Leanne M. Mauriello.

$\mathrm{Na}$ análise do grau de relevância dos periódicos, foi realizada uma estimativa daqueles que possuíam maiores publicações dentre os escolhidos no Portfólio Bibliográfico. Neste caso, o periódico Preventive Medicine foi o que obteve maior relevância, com dois artigos publicados.

No intuito de fundamentar o grau de relevância, foi descrito, também, o fator de impacto dos periódicos que variaram entre 0,609 , neste caso o The Journal of School Nursing,até 3.580 relativo ao periódico International Journal of Behavioral Nutrition and Physical Activity.

Ao estimar as palavras-chave mais utilizadas entre os artigos que compuseram o Portfólio, encontrou-se três que foram mais frequentes, sendo elas: Adolescents, Motor Activity e Obesity/Overweight.

Fato interessante é que em apenas um foi possível identificar a palavra-chave School-based, demonstrando que mesmos que os artigos incluídos no Portfólio estavam alinhados ao tema de pesquisa, somente após a leitura completa e detalhada, foi possível identificar as intervenções de base escolar utilizando estratégias do Modelo Transteorético.

A análise sistêmica consistiu na interpretação dos artigos pertencentes ao Portfólio Bibliográfico, de acordo com as lentes/critérios definidos a priori pelos pesquisadores. A definição destes critérios/lentes levaram em consideração a filiação teórica e a visão de mundo dos interessados na análise ${ }^{11}$. Para o presente estudo foram utilizados as seguintes lentes ou critérios a fim de orientar a análise sistêmica no tópico de discussão, conforme Tabela 2.

Para demonstrar os estudos de intervenção que utilizaram o modelo transteorético, foi realizado um sumário com seus principais achados e, em seguida, uma análise sistêmica dos mesmos. No quadro 1 encontra-se o sumário da análise sistêmica dos artigos selecionados. 
Tabela 1 - Portfólio Bibliográfico.

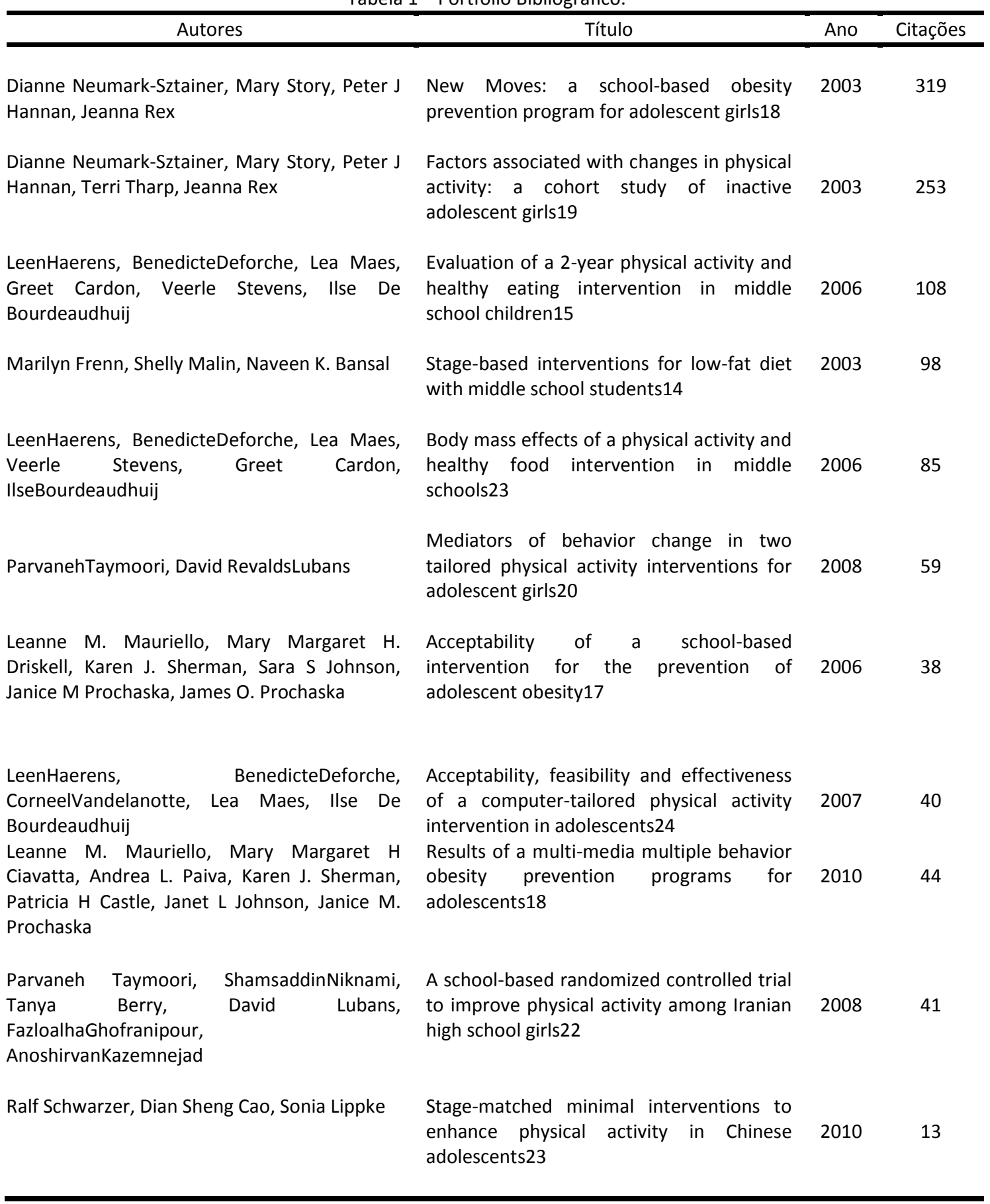

Tabela 2 - Lentes/critérios utilizados na análise sistêmica.

\begin{tabular}{ll}
\hline Lente/Critério & Caracterização e Abrangência \\
\hline Natureza & Qual a forma dos estudos que compõe o Portfólio Bibliográfico? \\
Abordagem & Os artigos apresentam uma abordagem quantitativa ou qualitativa? \\
Técnicas & São utilizadas técnicas estatísticas adequadas? \\
Variáveis & Quais são as outras variáveis investigadas nos artigos? \\
Conhecimento & Os artigos acrescentaram algum tipo de conhecimento inovador? \\
\hline
\end{tabular}

Fonte: Adaptado de Richartz et al ${ }^{12}$. 
Quadro 1 - Sumário de Estudos de Intervenção de base escolar utilizando os Estágios de Mudança de Comportamento.

\begin{tabular}{|c|c|c|c|c|c|c|}
\hline Autor(es) & Amostra & Delineamento & $\begin{array}{l}\text { Modelo de } \\
\text { Intervenção }\end{array}$ & $\begin{array}{l}\text { Tempo de } \\
\text { Intervenção }\end{array}$ & Resultados & Avaliação \\
\hline $\begin{array}{l}\text { Frenn et } \\
\text { al., } \\
200314\end{array}$ & $\begin{array}{l}182 \\
\text { escolare } \\
\text { s entre } \\
12 \text { e } 17 \\
\text { anos }\end{array}$ & $\begin{array}{l}\text { Pré e Pós- } \\
\text { intervenção } \\
\text { com Grupo } \\
\text { Controle }\end{array}$ & $\begin{array}{l}\text { Sessões de } \\
\text { informação sobre } \\
\text { atividade física e } \\
\text { alimentação }\end{array}$ & $\begin{array}{l}\text { Oito sessões } \\
\text { de } 45 \\
\text { minutos. }\end{array}$ & $\begin{array}{l}\text { Mudanças } \\
\text { significativas no } \\
\text { consumo de } \\
\text { gordura e nível de } \\
\text { atividade física em } \\
\text { relação ao grupo } \\
\text { controle }\end{array}$ & $\begin{array}{l}\text { Apesar das } \\
\text { melhorias } \\
\text { identificadas, há } \\
\text { necessidade de } \\
\text { aumentar o } \\
\text { número de } \\
\text { sessões e incluir } \\
\text { outras variáveis } \\
\text { para confirmar } \\
\text { os achados. }\end{array}$ \\
\hline $\begin{array}{l}\text { Haerens } \\
\text { et al., } \\
200615\end{array}$ & $\begin{array}{l}2.287 \\
\text { escolare } \\
\text { s entre } \\
14 \text { e } 18 \\
\text { anos }\end{array}$ & $\begin{array}{l}\text { Pré e Pós- } \\
\text { intervenção } \\
\text { com Grupo } \\
\text { Controle e } \\
\text { Drop-out }\end{array}$ & $\begin{array}{l}\text { Aulas de Educação } \\
\text { Física, Suporte } \\
\text { Social, } \\
\text { Comportamental } \\
\text { (EMC) e } \\
\text { computadores } \\
\text { pessoais }\end{array}$ & Dois anos & $\begin{array}{l}\text { Efeitos positivos } \\
\text { para atividade física } \\
\text { em ambos os sexos } \\
\text { e para consumo de } \\
\text { gorduras nas moças. }\end{array}$ & $\begin{array}{l}\text { Suporte de } \\
\text { parentes parece } \\
\text { não interferir na } \\
\text { intervenção e o } \\
\text { uso de } \\
\text { computadores } \\
\text { pode ser uma } \\
\text { ferramenta } \\
\text { importante. No } \\
\text { entanto, carece } \\
\text { de outros } \\
\text { estudos. }\end{array}$ \\
\hline $\begin{array}{l}\text { Haerens } \\
\text { et al., } \\
200724\end{array}$ & $\begin{array}{l}\text { Duas } \\
\text { salas de } \\
\text { aula de } \\
\text { escolare } \\
\text { s entre } \\
13 \text { e } 14 \\
\text { anos }\end{array}$ & $\begin{array}{l}\text { Pré e Pós- } \\
\text { intervenção } \\
\text { com Grupo } \\
\text { Controle }\end{array}$ & $\begin{array}{l}\text { Atividades } \\
\text { desenvolvidas no } \\
\text { computador para } \\
\text { atividade física }\end{array}$ & Três meses & $\begin{array}{l}\text { Melhoria nos níveis } \\
\text { de atividade física } \\
\text { na escola,em média, } \\
\text { de } 25 \text { min/semana, } \\
\text { mas não efetivo } \\
\text { para todas as } \\
\text { atividades no tempo } \\
\text { livre }\end{array}$ & $\begin{array}{l}\text { O uso do } \\
\text { computador } \\
\text { apresenta boa } \\
\text { aceitabilidade, } \\
\text { no entanto não } \\
\text { deve ser tão } \\
\text { longo o seu uso. }\end{array}$ \\
\hline $\begin{array}{l}\text { Mauriello } \\
\text { et al., } \\
200617\end{array}$ & $\begin{array}{l}45 \\
\text { escolare } \\
\text { s de } \\
\text { duas } \\
\text { escolas }\end{array}$ & $\begin{array}{l}\text { Estudo piloto } \\
\text { para um } \\
\text { programa de } \\
\text { intervenção }\end{array}$ & $\begin{array}{l}\text { Avaliar a } \\
\text { aceitabilidade de } \\
\text { uma intervenção } \\
\text { com o uso de } \\
\text { computadores }\end{array}$ & $\begin{array}{l}\text { Quatro } \\
\text { sessões de } \\
\text { aulas }\end{array}$ & $\begin{array}{l}\text { Boa aceitabilidade } \\
\text { do programa para } \\
\text { mudanças de } \\
\text { hábitos }\end{array}$ & $\begin{array}{l}\text { O programa } \\
\text { testado pode } \\
\text { ser utilizado em } \\
\text { estudantes } \\
\text { seguindo as } \\
\text { premissas do } \\
\text { modelo } \\
\text { transteorético. }\end{array}$ \\
\hline $\begin{array}{l}\text { Mauriello } \\
\text { et al., } \\
201018 .\end{array}$ & $\begin{array}{l}1800 \\
\text { escolare } \\
\text { s de } \\
\text { quatro } \\
\text { estados } \\
\text { america } \\
\text { nos }\end{array}$ & $\begin{array}{l}\text { Grupos } \\
\text { randomizados } \\
\text { com grupo } \\
\text { controle e } \\
\text { intervenção }\end{array}$ & $\begin{array}{l}\text { Informações } \\
\text { disponibilizadas } \\
\text { nas escolas com } \\
\text { uso do } \\
\text { computador }\end{array}$ & $\begin{array}{l}\text { Doze meses } \\
\text { com folow- } \\
\text { up }\end{array}$ & $\begin{array}{l}\text { Mudança dos } \\
\text { estágios de ação e } \\
\text { manutenção } \\
\text { significativas em } \\
\text { relação ao grupo } \\
\text { controle }\end{array}$ & $\begin{array}{l}\text { As modificações } \\
\text { em curto prazo } \\
\text { parecem ser } \\
\text { mais eficientes } \\
\text { com os estágios } \\
\text { de mudança de } \\
\text { comportamento } \\
\text {. }\end{array}$ \\
\hline
\end{tabular}

Continua... 
... continuação.

\begin{tabular}{|c|c|c|c|c|c|c|}
\hline $\begin{array}{l}\text { Neumark- } \\
\text { Sztainer } \\
\text { et al., } \\
200319\end{array}$ & $\begin{array}{l}221 \\
\text { moças } \\
\text { entre } 14 \\
\text { e } 18 \\
\text { anos de } \\
\text { idade }\end{array}$ & $\begin{array}{l}\text { Pré e Pós- } \\
\text { intervenção } \\
\text { com grupo } \\
\text { controle e } \\
\text { follow-up. }\end{array}$ & $\begin{array}{l}\text { Aulas de educação } \\
\text { física, Suporte } \\
\text { Social } \\
\text { Comportamental } \\
\text { (EMC) }\end{array}$ & Seis meses & $\begin{array}{l}\text { Moças do grupo de } \\
\text { intervenção } \\
\text { progrediram nos } \\
\text { EMC desde } \\
\text { baseline até } \\
\text { follow-up }\end{array}$ & $\begin{array}{l}\text { Apesar das } \\
\text { melhorias do } \\
\text { comportamento } \\
\text { de atividade } \\
\text { física } \\
\text { alimentação, } \\
\text { não foram } \\
\text { observadas } \\
\text { diferenças na } \\
\text { maioria das } \\
\text { variáveis entre o } \\
\text { baseline e pós- } \\
\text { intervenção. }\end{array}$ \\
\hline $\begin{array}{l}\text { Neumark- } \\
\text { Sztaine r } \\
\text { et al., } \\
200320\end{array}$ & $\begin{array}{l}201 \\
\text { moças } \\
\text { inativas } \\
\text { fisicame } \\
\text { nte } \\
\text { entre } 14 \\
\text { e } \quad 18 \\
\text { anos de } \\
\text { idade }\end{array}$ & $\begin{array}{l}\text { Avaliação de } \\
\text { intervenção } \\
\text { (transversal) }\end{array}$ & $\begin{array}{l}\text { Aulas de Educação } \\
\text { Física, Suporte } \\
\text { Social } \\
\text { Comportamental } \\
\text { (EMC) }\end{array}$ & Oito meses & $\begin{array}{l}\text { Duas evidências } \\
\text { fortes no aumento } \\
\text { do nível de } \\
\text { atividade física } \\
\text { foram o tempo e o } \\
\text { suporte social } \\
\text { (pares parentes e } \\
\text { professores) }\end{array}$ & $\begin{array}{l}\text { A intervenção } \\
\text { parece melhorar } \\
\text { os níveis de } \\
\text { atividade física } \\
\text { sempre quando } \\
\text { aumenta } \\
\text { suporte } \\
\text { amigos, } \\
\text { professores e } \\
\text { pais. }\end{array}$ \\
\hline $\begin{array}{l}\text { Taymoori } \\
\& \text { Lubans, } \\
200821\end{array}$ & $\begin{array}{l}161 \\
\text { escolare } \\
\text { s moças }\end{array}$ & $\begin{array}{l}\text { Grupos } \\
\text { randomizados } \\
\text {, com controle } \\
\text { e dois } \\
\text { intervenção } \\
\text { verificando os } \\
\text { mediadores } \\
\text { para atividade } \\
\text { física }\end{array}$ & $\begin{array}{lr}\text { Sessões } & \text { de } \\
\text { informação sobre } \\
\text { atividade física e } \\
\text { aulas } \quad \text { com } \\
\text { participação das } \\
\text { mães }\end{array}$ & $\begin{array}{l}\text { Seis meses } \\
\text { com folow- } \\
\text { up }\end{array}$ & $\begin{array}{l}\text { Positivo em um } \\
\text { grupo para barreiras } \\
\text { percebidas e auto- } \\
\text { eficácia e, no outro, } \\
\text { para planejamento } \\
\text { de atividade física } \\
\text { como mediadores }\end{array}$ & $\begin{array}{l}\text { Os dois modelos } \\
\text { de intervenção } \\
\text { promovem } \\
\text { benefícios } \\
\text { positivos } \\
\text { quando } \\
\text { observados os } \\
\text { aspectos } \\
\text { comportamenta } \\
\text { is. }\end{array}$ \\
\hline $\begin{array}{l}\text { Taymoori } \\
\text { et al., } \\
200822\end{array}$ & $\begin{array}{l}161 \\
\text { escolare } \\
\text { s moças }\end{array}$ & $\begin{array}{l}\text { Grupos } \\
\text { randomizados } \\
\text {, com controle } \\
\text { e dois } \\
\text { intervenção }\end{array}$ & $\begin{array}{lr}\text { Sessões } & \text { com } \\
\text { informações } & \\
\text { utilizando } & \text { o } \\
\text { modelo } & \\
\text { transteorético e } \\
\begin{array}{l}\text { educação em } \\
\text { saúde }\end{array}\end{array}$ & $\begin{array}{l}\text { Seis meses } \\
\text { com folow- } \\
\text { up }\end{array}$ & $\begin{array}{lr}\text { Aumento } & \text { na } \\
\text { percepção } & \text { para } \\
\text { participação } & \text { em } \\
\text { atividades física nos } & \text { no } \\
\text { dois grupos em } \\
\text { relação ao controle }\end{array}$ & $\begin{array}{lr}0 & \text { estudo } \\
\text { demonstra as } \\
\text { primeiras } \\
\text { evidências de } \\
\text { modelos } \\
\text { combinados de } \\
\text { intervenção no } \\
\text { aumento da } \\
\text { prática } \\
\text { atividade física. }\end{array}$ \\
\hline $\begin{array}{l}\text { Schwarzer } \\
\text { et al., } \\
201023\end{array}$ & $\begin{array}{l}15 \\
\text { escolas } \\
\text { com } \\
\text { escolare } \\
\text { s entre } \\
14 \text { e } 18 \\
\text { anos }\end{array}$ & $\begin{array}{l}\text { Dois grupos } \\
\text { de } \\
\text { intervenção e } \\
\text { um grupo } \\
\text { controle }\end{array}$ & $\begin{array}{l}\text { Informações } \\
\text { impressas sobre } \\
\text { atividade física e } \\
\text { uma hora de } \\
\text { reunião }\end{array}$ & $\begin{array}{l}\text { Quatro } \\
\text { semanas }\end{array}$ & $\begin{array}{l}\text { Aumento na } \\
\text { participação em } \\
\text { atividade física nos } \\
\text { estágios inferiores e } \\
\text { manutenção do } \\
\text { nível nos estágios } \\
\text { superiores }\end{array}$ & $\begin{array}{l}\text { Este modelo } \\
\text { parece não } \\
\text { influenciar } \\
\text { aqueles que não } \\
\text { estavam } \\
\text { dispostos a } \\
\text { mudar o com- } \\
\text { portamento. } \\
\text { As mudanças } \\
\text { com o modelo } \\
\text { de suporte } \\
\text { parental } \\
\text { apresenta } \\
\text { melhores } \\
\text { resultados em } \\
\text { meninas. }\end{array}$ \\
\hline
\end{tabular}




\section{Discussão}

Seguindo a analise sistêmica, a discussão foi realizada com base nos critérios adotados a priori.

Em estudo realizado nos EUA ${ }^{14}$, os autores realizaram uma intervenção do tipo quaseexperimental, baseada nos modelos comportamentais de Promoção da Saúde e Estágio de Mudança de Comportamento. Teve o objetivo de diminuir o consumo de alimentos gordurosos e aumentar a prática de atividade física em escolares do ensino médio, de Milwaukee/EUA, de famílias de classe baixa e diferentes etnias, do grupo intervenção $(n=60)$ em relação ao controle $(n=57)$. Não houve diferenças estatísticas entre os grupos em relação aos aspectos sociodemográficos, consumo de gorduras e atividade física antes da intervenção. A intervenção se mostrou efetiva quando analisada a progressão dos estágios de mudança de comportamento do grupo intervenção, tendo a intenção de diminuir a ingestão de gorduras e aumentar a prática de atividade física.

Neste modelo de intervenção, as mudanças em curto prazo, com o suporte parental, parecem ser efetivas, já que foram apenas quatro sessões de 45 minutos (grupo intervenção) de atividades baseadas na combinação das teorias. Para os autores, o fato da intervenção oferecer as estratégias de mudanças de maneira quase personalizada e com um grupo pequeno, fez com que houvesse a progressão nos estágios, mesmo com poucas horas de intervenção. Mesmo não tendo sido aleatória a distribuição dos grupos, os resultados devem ser considerados para futuros estudos de replicabilidade. Ainda, para os autores, o fato de a intervenção oferecer as estratégias de mudanças de maneira quase personalizada e com um grupo pequeno, fez com que houvesse a progressão nos estágios, mesmo com poucas horas de intervenção.

No entanto, os autores relatam que por se tratar de um estudo de curta duração, as premissas de causalidade não podem ser aplicadas, necessitando de estudos com maior tempo de duração (longitudinais). Acrescentam que mesmo não tendo sido aleatória a distribuição dos grupos, os resultados devem ser considerados para futuros estudos de replicabilidade. Ao analisar este estudo, observase que existe, ainda, uma limitação metodológica, já que não houve uma aleatorização dos grupos após a coleta no baseline, não apresentando os resultados de maneira clara e as tabelas são confusas. Contudo, pontos positivos devem ser destacados, como: seleção de instrumentos validados, utilização de teorias combinadas e estratégia de mudança aplicada quase individualmente.

Intervenção de longa duração (dois anos), realizada na Bélgica ${ }^{15}$, com 2.840 escolares com média de idade de 13 anos, os autores buscaram observar os efeitos sobre o nível de atividade física e hábitos alimentares. Utilizaram as estratégias do EMC, sortearam 15 escolas, das quais: cinco escolas utilizaram o suporte parental, cinco sem suporte e cinco como controle. Realizaram, ainda, um levantamento do tipo drop-out após os dois anos de intervenção. Além dos instrumentos do tipo questionário, foi utilizada, em uma sub-amostra, a acelerometria. Para as estratégias da intervenção foi criado um grupo de trabalho envolvendo os professores, direção da escola, além dos pais de um dos grupos de intervenção. Dentre os vários resultados encontrados, os autores destacaram que houve efeito positivo para o nível de atividade física entre os dois sexos e diminuição significativa do consumo de gorduras entre as moças. Neste estudo, o suporte parental não demonstrou influenciar na efetividade da intervenção. Quando analisados os dados da acelerometria, observou-se um incremento na participação em atividades moderadas a vigorosas entre os garotos da sub-amostra e uma subsequente estabilização após os dois anos de intervenção.

Mesmo os autores apresentando algumas limitações como: uma taxa de abandono de $25 \%$ e, como o nível de atividade física e de hábitos alimentares foi medido através de questionário, erros podem ter sido reportados. O estudo apresenta robustez, o desenho da intervenção é bem explicado, os dados são bem apresentados em tabelas de fácil entendimento.

Em outro estudo 16, com a mesma amostra do anterior, os autores buscaram avaliar aos efeitos da intervenção sobre o Índice de Massa Corporal (IMC), além do nível de atividade física e de hábitos alimentares em escolares. Em relação ao nível de atividade física e de hábitos alimentares, os dados foram apresentados anteriormente. O estudo utilizou a medida de IMC em $\mathrm{Kg} / \mathrm{m} 2$, além do Escore $Z$, encontrando efeitos positivos para as moças em relação aos dois indicadores de estado nutricional. Evidenciou que esta melhoria aconteceu, principalmente, no grupo que teve o suporte parental. Entre os rapazes não foram observadas diferenças após a intervenção. O grupo de 
trabalho formado por professores, diretores e pais recebeu mídias e acesso à internet, com materiais que reforçaram as estratégias de intervenção, além de avaliar, ao final de cada ano, com os diretores, qual o nível de implementação das estratégias em cada escola. Quando ajustado pelo nível de implementação, os resultados do Escore $Z$ para $O$ IMC demonstraram que houve um aumento menor do índice em escolas com médio e alto nível de implementação. Os autores apontaram que as diferenças entre os sexos em relação aos resultados do estado nutricional podem estar relacionadas ao fato das estratégias de intervenção ser de forma generalizada, necessitando, em determinados momentos, de um direcionamento das informações de forma específica a cada sexo.

Em estudo piloto para testar a aceitabilidade de uma intervenção para modificação do comportamento relacionado a atividade física, hábitos alimentares e tempo assistindo $\mathrm{TV}^{17}$, os autores utilizaram como estratégia o uso do computador em sala de aula para dar suporte à intervenção. Este estudo foi realizado nos EUA, com uma amostra de 45 estudantes e utilizou as estratégias do Estágio de Mudança de Comportamento através de um instrumento com 16 itens, como forma de implementar o programa Health in Motion. Este programa foi baseado na prevenção da obesidade e na mudança de comportamento acerca da atividade física, hábitos alimentares e comportamento sedentário. O programa possui a as seguintes características: conscientização, decisão, autoeficácia, processo de mudança e estratégias. Para cada etapa das características, existiam informações gerais e específicas referenciadas em qual estágio de mudança de comportamento se encontrava a amostra. $\mathrm{O}$ teste piloto mostrou que $97 \%$ dos escolares declararam que iriam aderir ao programa, $88,9 \%$ acharam as questões de fácil entendimento e, principalmente, que $80 \%$ indicou que o programa é apropriado para a sua faixa etária. Os autores destacaram que $64,4 \%$ dos escolares informaram que o programa pode fazer com que eles se tornem mais saudáveis e que o programa de computador necessita de pouco treinamento, facilitando a adoção das estratégias e a mudança do comportamento.

Pode-se destacar, neste estudo, a preocupação de se testar todas as características de um programa de intervenção antes de implementá-lo, em especial, a qualidade das informações que serão transmitidas às escolas que adotarem este programa. O texto do estudo é de fácil compreensão, os dados estão bem visualizados e destaca-se o fato de todas as etapas do programa Health in Motion encontrarem-se descritas no artigo, facilitando sua replicabilidade.

Intervenção em 1.800 escolares de quatro estados dos EUA18, com o objetivo de avaliar os efeitos do Health in Motion nas variáveis: nível de atividade física, consumo de frutas e verduras e tempo assistindo TV. O desenho da intervenção previu dois grupos (intervenção $x$ controle), avaliando-os em momentos distintos (Baseline, um mês, dois meses, seis meses e 12 meses).Os escolares recebiam informações em três momentos distintos(Baseline, um e dois meses) e, após o sexto e décimo segundo mês, realizavam um levantamento do tipo follow-up. As estratégias da intervenção foram computadores com um software desenvolvido especialmente para o estudo. Os escolares utilizavam o computador para receber as informações necessárias de acordo com o momento de seu estágio de mudança de comportamento.

Os resultados demonstraram que, para atividade física, houve um aumento significativo do número de dias em atividades com duração de 60 min., após dois meses de intervenção. 0 consumo de frutas e verduras aumentou no número de porções diárias para dois, seis e 12 meses (follow-up) após a intervenção. Para o tempo assistindo TV, não houve diferença. Para os estágios de mudança de comportamento houve maior progressão entre os EMC de Ação e Manutenção entre os participantes do grupo intervenção após dois meses (28,5\% para $14,4 \%$ $\mathrm{OR}=2,10)$. Para o consumo de frutas e verduras de $35,5 \%$ para $12,7 \%(\mathrm{OR}=2,53)$ após dois meses e de $29,6 \%$ para $10,4 \%$ após seis meses. No entanto, para o tempo assistindo TV não houve diferença entre os grupos após a intervenção, porém os autores relataram que houve diferença quando analisados os dados do follow-up (seis e 12 meses) em relação ao segundo mês de intervenção.

Os autores alertam que este efeito pode não ser das estratégias de intervenção já que houve, também, certas melhorias nos indicadores do grupo controle após seis e 12 meses. Por fim, destacaram que não foi possível verificar diferenças entre os grupos quando analisados os dados do estado nutricional. Apontou, ainda, a principal limitação do estudo como: diferenças positivas de algumas variáveis no grupo controle, que pode estar relacionado ao fato deles 
responderem por quatro momentos distintos 0 instrumento de pesquisa, o que poderia influenciar em algumas mudanças do comportamento como um mini-tratamento.

O estudo apresenta dados importantes sobre as estratégias de intervenção, a leitura é de fácil compreensão, os dados são descritos em tabelas claras e destaca a importância de intervenções de base escolar em que haja aceitabilidade e facilidade na disseminação das informações, testadas anteriormente em estudo piloto.

Estudo piloto $^{19}$, os autores selecionaram moças em seis escolas devidamente randomizadas, nos EUA. Realizaram três coletas distintas (baseline, pós-intervenção e follow-up), tendo a intervenção duração de seis meses. Avaliaram: facilidade de entendimento da intervenção; medida de satisfação entre as moças, diretores, professores e pais; sugestões para modificação da intervenção e efeitos em curto prazo. Para os professores e diretores, a intervenção era de fácil aceitação, possível de ser implementada em longo prazo e, principalmente, a utilização de diários, fitas elásticas, acesso a centros de treinamento e pedômetro, incentivaram a participação das moças. Quando avaliado o questionário dos pais, $92 \%$ expressaram entusiasmo com o programa, $90 \%$ recomendariam a outros pais e outras moças e $100 \%$ gostariam que a intervenção fosse realizada novamente no ano seguinte. $\mathrm{Na}$ avaliação das moças, 91\% estavam satisfeitas de forma geral com a intervenção, $85 \%$ com as estratégias para atividade física, $89 \%$ para nutrição e $86 \%$ para suporte social. Ainda, foi criado um grupo de 30 moças para avaliar, de forma qualitativa, as estratégias da intervenção. Foram encorajadas a participar mediante pagamento de US\$ 25,00 e as respostas mais frequentes foram em relação ao controle de peso e imagem corporal.

Os efeitos da intervenção mostraram que as moças progrediram apenas nos EMC para a atividade física em relação ao grupo controle no follow-up, sendo que nas outras variáveis não houve mudanças significativas. Os autores indicaram que o programa de intervenção pode ser implementado nas escolas devido à excelente aceitabilidade das moças, pais, diretores e professores; no entanto, devem ser consideradas outras questões para verificar mudanças significativas nas variáveis de desfecho. Este estudo apresenta dados importantes para avaliação de um programa, principalmente ao utilizar instrumentos qualitativos, entrevista com os pais, professores e diretores. Os dados são de fácil entendimento, a metodologia bem descrita e os resultados bem apresentados.

Em outro estudo ${ }^{20}$, os autores observaram os efeitos de uma intervenção baseada no programa New Moves e os fatores associados às mudanças para atividade física para moças. A intervenção teve a duração de oito meses, sendo realizada em seis escolas nos EUA, em que as estratégias da intervenção foram: informações através de um guia sobre atividade física, hábitos alimentares, controle de peso, além de suporte social de professores, pais e diretores. Os resultados demonstraram, através de análise de regressão múltipla, que após a aplicação de vários modelos, as mudanças no nível de atividade física associaram-se fortemente com as restrições de tempo (inversamente), suporte social e parental para atividade física, além de auto-eficácia (positivamente). Destacaram que estes fatores sofrem grandes influências dos diferentes domínios resultantes da Teoria Social Cognitiva (comportamento, socioambiental e pessoal, respectivamente).

Sugerem que, em relação às restrições de tempo, as moças sejam estimuladas a diminuir o tempo assistindo TV, aumentando o tempo da prática de atividades físicas. Para o suporte social e parental, os pais e professores devem continuar estimulando a prática e, principalmente, os pares, já que nesta idade o suporte de amigos parece ser mais influente. Por fim, a associação com a auto-eficácia sugere que devem ser abordados os aspectos de autoimagem e a necessidade de suporte para melhoria nas habilidades de se praticar atividades físicas, especialmente os esportes que são mais frequentes nesta idade. Os autores apontam que uma das limitações do estudo foi o fato da análise se basear nas medidas do nível de atividade física auto referidas. 0 estudo apresenta diversas informações importantes acerca do modelo de intervenção (instrumento, medidas e análise estatística), a metodologia é de fácil compreensão e os resultados encontram-se todos bem descritos. A principal limitação já foi descrita pelos próprios autores.

Em estudo realizado com moças no Irã $^{21}$, os autores buscaram evidenciar os mediadores para a mudança de comportamento para atividade física em duas intervenções adaptadas. Para tanto, utilizaram um grupo controle, um grupo de intervenção baseado no modelo de Promoção da Saúde e outro baseado nos Estágios de Mudança de Comportamento. As intervenções tiveram seis meses de duração, com estratégias 
de atividades em grupo, conselhos individuais e reforço das mensagens por telefone. Utilizaramse, para os dois modelos de intervenção, os possíveis mediadores encontrados na literatura: benefícios e barreiras percebidas; auto-eficácia; exposição aos modelos; suporte social e interpessoal; planejamento e nível de condicionamento. Várias hipóteses foram levantadas como os mais fortes mediadores para as mudanças no nível de atividade física após a intervenção, independente do modelo, tais como: comportamentais, cognitivos e interpessoais.

Quando analisada a intervenção baseada nos EMC, os autores relataram que as variáveis que tiveram o maior poder de mediação foram: benefícios percebidos, barreiras percebidas, auto-eficácia e compromisso para planejar a ação. Para a intervenção baseada no modelo de Promoção da Saúde, as variáveis foram: autoeficácia e compromisso para planejar a ação. Ambas as intervenções produziram efeitos positivos em relação ao aumento do nível de atividade física em relação ao grupo controle. Destacaram que, quando analisados os fatores interpessoais, nove deles satisfizeram o modelo de mediação, sugerindo um forte poder explicativo. O mesmo aconteceu com os fatores cognitivos e comportamentais em menor proporção. Como contribuições do estudo, afirmaram que, para as intervenções comportamentais, há necessidade de se preconizar mais organização, avaliação e implementação das atividades. Por fim, indicaram que a intervenção teve um forte impacto nas mudanças de comportamentos nas moças.

Em outro estudo ${ }^{22}$, utilizando a mesma amostra e metodologia descrita anteriormente, buscaram evidenciar os efeitos das intervenções sobre o nível de atividade física nas moças, avaliando, desta vez, as variáveis de tempo de prática. $O$ estudo demonstrou que os modelos modificaram significativamente os níveis de atividade física em relação ao controle e ao baseline, aumentando em quase $56 \mathrm{~min} / \mathrm{dia}$. Ao avaliar o follow-up, não foram observadas diferenças entre os grupos. Os autores revelaram que os dois modelos promovem efeitos positivos, no entanto, devem ser considerados aspectos organizacionais e não somente comportamentais e cognitivos, como: mudança de estrutura física, medida objetiva do nível de atividade física, controle do condicionamento e planejamento das atividades baseadas no nível de habilidades.

No estudo realizado na China, com escolares entre 12 e 17 anos $^{23}$ dividiram os escolares em três grupos distintos, sendo dois intervenção e um controle. A intervenção consistia em oferecer aos escolares dos grupos de intervenção apenas uma hora de informações. Posteriormente, os grupos foram divididos de acordo com os estágios de comportamento que se encontravam. Os grupos foram divididos em préintenção, intenção e ação e a cada grupo foi oferecido um tipo de estratégia. A intervenção teve quatro semanas de duração. Os autores apontaram que a intervenção curta e com informações específicas, a cada estágio, parece ser mais efetiva para a mudança do comportamento naqueles que se encontravam na pré-intenção e intenção. Quando analisados os grupos de ação, houve recaída em relação aos outros. As estratégias de comunicação e planejamento das ações foram mais efetivas entre os estágios inferiores em relação ao grupo controle. Ao observar os efeitos das estratégias para o grupo de ação, houve recaída em relação ao grupo controle.

A avaliação dos autores é que os indivíduos que já possuem um nível elevado de atividade física, quando expostos às informações que já conhecem, parece ocasionar certo acomodamento do comportamento e,às vezes, ocorre a recaída. Mas apontam que o estudo é inovador por ser o primeiro na China e por incorporar estratégias específicas para cada estágio de mudança e, principalmente, pelo avanço daqueles que mais necessitam delas. 0 estudo foi bem conduzido, introduzindo uma estratégia de intervenção de apenas uma hora, dividindo os grupos de acordo com cada estágio, mesmo sendo uma amostra relativamente grande.

Estudo realizado na Belgica $^{24}$ teve como objetivo de verificar a aceitabilidade, viabilidade e efetividade de um programa de intervenção utilizando computadores (programa) em sala de aula. Os adolescentes tinham em média 13 anos, e foram divididos em grupos de intervenção e controle. A intervenção durou três meses e utilizaram-se estratégias do MT para a atividade física. Os resultados apontaram para uma boa aceitabilidade da proposta, os adolescentes identificaram como positiva a viabilidade da intervenção, principalmente, em relação ao uso do computador e as informações contidas no programa. Em relação aos efeitos sobre a atividade física, os adolescentes descreveram que as informações no programa não eram tão simples e eram longas, dificultando a viabilidade. No entanto, houve aumento significativo na 
participação de atividades físicas no ambiente escolar, do grupo de intervenção em relação ao controle. Apontaram que não houve efeitos significativos nas demais dimensões da atividade física.

Os autores sugerem que os efeitos da intervenção sobre a atividade física global, poderiam ser alcançados, se a intervenção fosse superior a três meses, e ajustados ao problema identificado no questionário prévio. Mas ressaltaram uma discreta, porém, significante melhoria em relação às atividades no ambiente escolar.

O artigo possui uma análise robusta, é de fácil entendimento, pode ser replicado sem dificuldade.

\section{Conclusões}

O presente estudo identificou onze artigos que atenderam aos critérios adotados previamente de acordo com o método. Em relação ao tempo de publicação dos artigos, a maioria havia sido publicado há mais de cinco anos e apenas um periódico havia publicado mais de um artigo sobre o tema proposto.

Para estimativa dos autores mais citados, uma das autoras obteve as maiores quantidades de citações, sendo quase maior que de todos os outros que compuseram o Portfólio, o que se deve ao fato de maior tempo de publicação. Os descritores mais frequentes estavam, em parte, de acordo com os utilizados no processo inicial de confecção do Banco de Artigos Brutos, porém foi observado que alguns descritores, apesar de serem apresentados nos títulos e resumos dos artigos, não faziam parte de descritores na área de saúde. Ao estimar o fator de impacto dos periódicos, observou-se que aquele que tinha o maior índice, não foi o que alcançou maior número de citações.

$\mathrm{Na}$ análise sistêmica, ao avaliar os estudos e com base nas lentes/critérios determinados a priori, a maioria foi de natureza empírica, abordagem quantitativa, com analise estatística adequada, além da atividade física, alguns analisaram outras variáveis e utilizaram as estratégias do Modelo Transteorético isolada ou combinada.

Não houve unanimidade em relação aos resultados. A utilização das estratégias do Modelo Transteorético demonstrou que, em alguns estudos, houve mudanças importantes nos comportamentos dos adolescentes em relação à variável atividade física.
Grande parte dos autores indicou que o fato de usarem a medida auto-referida da atividade física como desfecho principal, pode ser a maior limitação dos estudos. Apesar das abordagens serem inovadoras na maioria dos estudos, os autores sugerem que devam ser realizados novos estudos, com amostras maiores e instrumentos mais precisos para medida das variáveis de interesse.

\section{Referências}

1. World Health Organization. Global Strategy on Diet, Physical Activity and Health. Physical Activity and Young People. Disponível: http://www.who.int/dietphysicalactivity/factshe et_young_people/en/. (acessado em 20 de Dezembro de 2015)

2. Prochaska JO, Redding CA, Evers KE. The transtheoretical model and stages of change. Health Behavior.2008; 97-110.

3. Timperio A. Salmon J. Ball K .Evidence based strategies to promote physical activity among children, adolescents and young adults: review and update. J of Sciand Med in Sport:,7 \{1): Supplement: 20-29, 2004.

4. Oliveira NKRD, Barros MVGD, Reis RS, Tassitano RM, Tenório MCM, Bezerra J, et al. Estágios de mudança de comportamento para a atividade física em adolescentes.Motriz Rev Educ Fís .2012;18(1):42-54.

5. Pelegrini A Silva, DAS Corseuil, HX Silva, JMFDL Petroski, EL. Estágios de mudança de comportamento relacionado à atividade física em adolescentes de uma capital brasileira. Motriz Rev Educ Fís. 2013;19(4):770-5.

6. Silva DAS, Pelegrini A, Grigollo LR, Silva $A F$, Petroski EL. Diferenças e similaridades dos estágios de mudança de comportamento para atividade física em adolescentes de duas áreas brasileiras. Rev Paul Pediatr. 2011;29(2):193-201. 7. Lonsdale $C$, Rosenkranz RR, Peralta LR, Bennie A, Fahey P, Lubans DR. A systematic review and meta-analysis of interventions designed to increase moderate-to-vigorous physical activity in school physical education lessons. Prev Med. 2013; 56(2):152-61.

8. Barboza CF, Monteiro SM, Barradas SC, Sarmiento OL, Rios $P$, Ramirez A, et al. Physical activity, nutrition and behavior change in Latin 
America: a systematic review. Global Health Promotion. 2013;20 (Suppl 4):65-81.

9. Ensslin L. ProKnow-C, Knowledge Development Process-Constructivist: processo técnico com patente de registro pendente junto ao INPI. Brasil; 2010.

10. Chaves LC, Ensslin L, Ensslin SR, Valmorbida SMI, Rosa FS.Sistemas de apoio à decisão: mapeamento e análise de conteúdo. Rev Eletr Ciênc Admin. 2013:12(1):6-22.

11. Vilela LO. Aplicação do ProKnow-C para seleção de um portfólio bibliográfico e análise bibliométrica sobre avaliação de desempenho da gestão do conhecimento. Rev Gestão Industrial.2012;8(1):76-92.

12. Richartz $F$, Borgert A, Ensslin SR. Comportamento dos custos: mapeamento e análise sistêmica das publicações internacionais.Sociedade, Contabilidade e Gestão. 2015;9(3):92-108.

13. Salvio, SC.Guia de utilização do EndNote Web.Campinas: Biblioteca IFGW ;2013.

14. Frenn M, Malin S, Bansal NK. Stagebased interventions for low-fat diet with middle school students. J Pediatr Nurs. 2003;18:36-45.

15. Haerens L, Deforche B, Maes L, Stevens V, Cardon G, DeBourdeaudhuijl. Evaluation of a 2-year physical activity and healthy eating intervention in middle school children. Health EducRes. 2006;21:911-21.

16. Haerens L, Deforche B, Maes L, Stevens V, Cardon G, De Bourdeaudhuij I. Body mass effects of a physical activity and healthy food intervention in middle schools. Obesity (Silver Spring). 2006;14:847-54.

17. Mauriello LM, Driskell MH, Sherman KJ, Johnson SJ, Prochaska JM, Prochaska JO. Acceptability of a school-based intervention for the prevention of adolescent obesity. J School Nurs. 2006;22(5):269-77.

18. Mauriello LM, Giavatta MMH, Paiva AL, Sherman KJ, Castle PH, Hohnson JL, et al. Results of a multi-media multiple behavior obesity prevention program for adolescents. Prev Med. 2010;51:451-6.

19. Neumark-Sztainer D, Story M, Hannan P, Stat M, Rex J. New moves: a school-based obesity prevention program for adolescent girls. Prev Med. 2003;37:41-53.
20. Neumark-Sztainer D, Story M, Hannan P, Tharp T, Rex J. Factors associated with changes in physical activity: a cohort study of inactive adolescent girls. Arch Pediatr Adolesc Med. 2003;157: 803-10.

21. Taymoori P, Lubans DR. Mediators of behavior change in two tailored physical activity interventions for adolescent girls. Psychol Sport Exerc. 2008;9:605-19.

22. Taymoori P, Niknami S, Berry T, Ghofranipour F, Kazemnejad A. A school-based randomized controlled trial to improve physical activity among Iranian high school girls. Int J Behav Nutr Phys. 2008; 5:18.

23. Schwarzer R, Cao DS, Lippke S. Stagematched minimal interventions to enhance physical activity in Chinese adolescents. J Adolesc Health, 2010; 47: 533-9.

24. Haerens L, Deforche B, Vandelanotte C, Maes L, De Bourdeaudhuij I. Acceptability, feasibility and effectiveness of a computertailored physical activity intervention in adolescents. Pat Educ and couns. 2007; 66(3): 303-310.

\section{Endereço para Correspondência}

Universidade Estadual do Sudoeste da Bahia UESB

Av. José Moreira Sobrinho, s/n - Jequiezinho Jequié (BA)

CEP.: 45206-510

e-mail: hlrmunaro@uesb.edu.br

Recebido em 08/06/2016

Aprovado em 06/04/2017

Publicado em 03/05/2017 